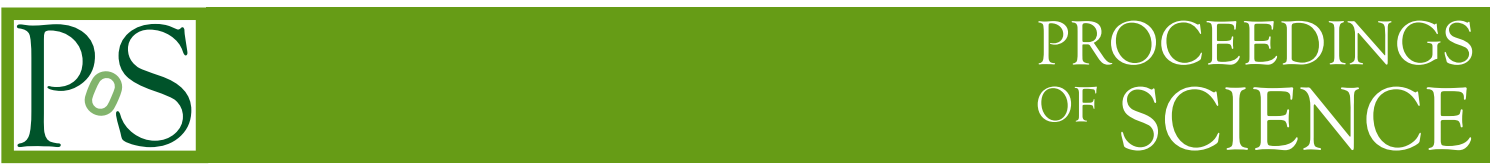

\title{
Collectivity of soft probes in small systems
}

\section{Tomasz Bold ${ }^{\dagger * *}$ on behalf of the ATLAS, CMS and ALICE Collaborations}

AGH University Of Science and Technology,

Mickiewicza 30, Krakow, Poland

E-mail: tomasz.bold@cern.ch

The relativistic collisions of heavy ions revealed the existence of a new, dense and hot state of matter, the quark-gluon plasma (QGP). Models assuming that after the collision, the QGP stage occurs and the QGP behaves like nearly perfect liquid are successful in describing collective behaviours observed experimentally. Prior to the LHC heavy-ion programme the collisions of smaller systems like proton-ion or proton-proton were thought to be unable to produce the QGP. Surprisingly, experimental results obtained by the LHC experiments indicated that some signatures that were attributed to the emergence of collectivity due to the QGP were also observed in collisions of smaller systems. The most striking are observation of long-range azimuthal correlations even in a relatively small multiplicity proton-proton collisions. The origin of those effects is at the moment an open question. Is a tiny droplet of QGP created on the way from the hard scatter to the final state or the signatures of collectivity can be explained without recalling to this phenomenon? This report discusses recently performed measurements of collectivity in small systems which aim at correlating soft probes with the presence of a hard process in the event.

The Ninth Annual Conference on Large Hadron Collider Physics - LHCP2021

7-12 June 2021

Online

\footnotetext{
*Speaker

$\dagger^{\dagger}$ This work was partly supported by the National Science Centre of Poland under grant number UMO2020/37/B/ST2/01043 and by PL-GRID infrastructure.
} 


\section{Introduction}

This report discusses experimental results on correlations of soft probes from ALICE [1], ATLAS [2], and CMS [3] experiments at the LHC.

One of the signatures of the quark-gluon plasma (QGP) is the observation of long-range correlations between particles produced in heavy-ion collisions. The most basic observable is the observation of the structure in two particle correlations (2PC). The correlation is obtained as a function $\Delta \phi$ and $\Delta \eta$ between particles of interest. Structures observed for small $\Delta \eta$ between the particles are determined by QCD di-jets and resonance decays (typically called non-flow). That structure quickly fades away when the $\Delta \eta$ is larger and another structure appears, the ridge. That long-range structure is an emanation of the existence of a global correlation that spans a wide rapidity range. The first observation of such structure in $p p$ events was done by CMS [4] in 2010. In retrospect, it was the first experimental sign that leads to the current debate about the origin of the long-range correlations in small system.

For quantitative measurement the $2 \mathrm{PC}$ correlation with the ridge are analysed by means of the Fourier decomposition. The yield is fitted with $Y(\Delta \phi)=C+G\left[1+2 \sum_{n} v_{n, n} \cos (n \Delta \phi)\right]$ where the $C, G$, and $v_{n, n}$ are free parameters. Thanks to $v_{n, n}$ factorisation property the single particle flow harmonic magnitude $v_{n}$ is obtained. The role of $C$ is to eliminate short-range correlations and it is obtained in different manners by LHC experiments. The ATLAS experiment uses a template obtained from correlations measured at low multiplicities or peripheral collisions while in case of ALICE and CMS the zero-at-minimum approach is used. Note that $v_{n}$ is an intensive variable and therefore relatively straightforward to compare across experiments and to the theory.

\section{A review of existing results on correlations in small systems}

The momentum dependence of $v_{n}$ for identified particles exhibits mass ordering in heavy-ion collisions. That is, the heavier the particle is the smaller the value of $v_{n}$ at low momentum is observed. The same observation was made by CMS [5] in $p+\mathrm{Pb}$ collisions as shown in Fig. 1 indicating a similar mechanism for momentum transfer in QGP. On the other hand the observed flow magnitude of the $D^{0}$ mesons is significantly smaller as compared to $\mathrm{Pb}+\mathrm{Pb}$ collisions and can not be explained just by the mass. It would indicate a weaker collectivity for charm quarks in the QGP.

The ATLAS experiment measured second flow harmonic of hadrons at high momentum [6] in $p+\mathrm{Pb}$. It is understood to originate from interactions of hard QCD probes with the QGP. A strong $v_{2}$ signal is present above $20 \mathrm{GeV}$ alike in $\mathrm{Pb}+\mathrm{Pb}$, as shown in the middle panel of Fig. 1, yet at the same time no jet modifications were observed in $p+\mathrm{Pb}$.

In $p p$ collisions the ridge was studied quantitatively by means of second flow harmonics magnitude and extracted values were found to be robustly different from 0 . Results from the ATLAS experiment [7] are shown in the right panel of Fig. 1. They were also found to be weakly dependent on event multiplicity and persist in events with the multiplicity as low as 20 charged particles.

Summarising, the possibility of creation of the QGP in collisions of small systems is supported by correlation measurements. However, not all the signatures are identical and theoretical expla- 

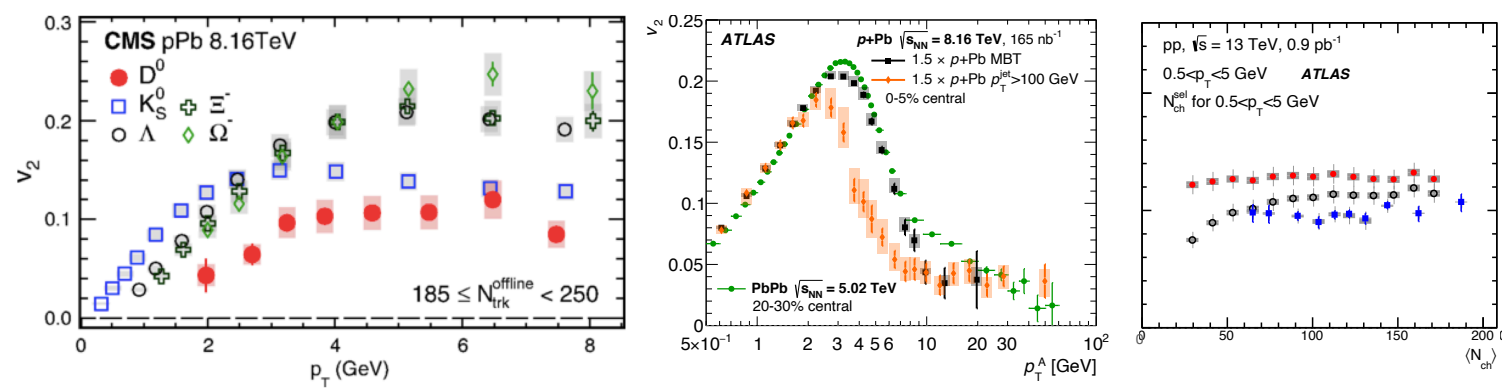

Figure 1: (left) The $v_{2}$ as a function of identified particle transverse momentum in $p+\mathrm{Pb}$ collisions by CMS [5]. (centre) The $v_{2}$ of charged hadrons in a broad $p_{\mathrm{T}}$ range in $p+\mathrm{Pb}$ and $\mathrm{Pb}+\mathrm{Pb}$ collisions by ATLAS [6]. (right) The $p_{\mathrm{T}}$-integrated $v_{2}$ measured in $p p$ collisions by ATLAS [7].

nations competing with the hydrodynamical evolving QGP are proposed. In such circumstances it is natural to exercise the technique known from ion collisions, that is, to bias the sample towards events with smaller or larger impact parameters and compare results to an inclusive events sample. The next section presents two such analyses performed by ATLAS and ALICE Collaborations.

\section{Sensitivity of the $v_{2}$ to the presence of the hard probe in the event}

The first analysis aiming at the selection of a sample with small impact parameters in $p p$ collisions was a measurement of correlations in $p p$ collisions tagged with a $Z$ boson [8]. The measurement indicated no significant difference between the $v_{2}$ in tagged and inclusive event samples. Neither differentially, as a function of charged hadrons $p_{T}$ nor in $p_{T}$-integrated $v_{2}$ in a broad range of event multiplicities.

A similar measurement, this time with QCD jets serving as the event hard scale is done by ATLAS [9]. Measuring correlations in the event sample without semi-hard (above $10 \mathrm{GeV}$ ) jets can indicate validity of models attributing observed correlations to arise from hard QCD processes. In the analysis, the events with jets are compared to an inclusive measurement and to the sample where no jet above the threshold was observed. In addition the correlation in the sample with jets is extracted eliminating particles in the vicinity of jets $(\Delta \eta<1)$. Altogether four correlations are obtained; an Inclusive one, inclusive one but with correlation extracted using particles away from jets, denoted as AllEvents, the sample without the jet denoted as NoJet, and the sample with jets, labeled WithJets, in which the correlation is extracted as in the AllEvents case. The $p_{\mathrm{T}}$-integrated and $p_{\mathrm{T}}$-differential $v_{2}$ for all those samples is shown in Fig. 2. The $v_{2}$ values of around $6 \%$ are observed across a broad multiplicity range and found to be nearly independent of the event category. In addition, the selection of the charged hadrons used for the construction of the correlation does not impact the result significantly. Similarly, only a small difference is observed in the differential result. The values obtained requiring higher or lower jet momentum are also consistent. If the observed correlations were to originate from semi-hard processes the $v_{2}$ in the sample without jets should be significantly different from the inclusive sample. Also, if the correlations were not global, the inclusion/exclusion of particles associated with the jet would have a significant impact on $v_{2}$. None of these is observed, which favours the picture in which the system evolution has a phase of collective expansion, that is the fluid-like QGP. 

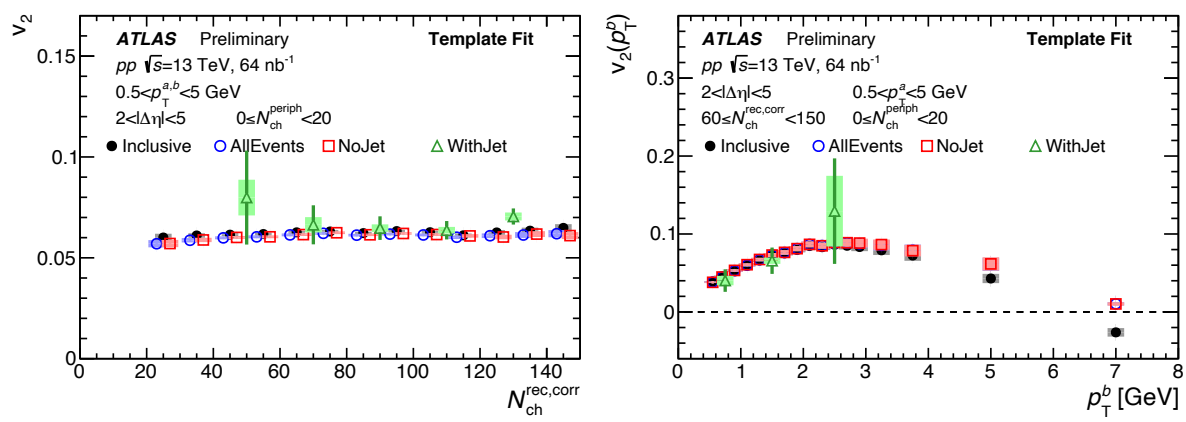

Figure 2: The $v_{2}$ in $p p$ collisions as a function of event multiplicity (left) and charged particle momenta (right) for samples including (Inclusive, All Events, WithJet) /excluding (NoJet) jets and including (Inclusive, NoJet)/excluding (AllEvents, WithJet) charged hadrons [9].

A similar measurement is performed by ALICE [10]. In this case the jet or a high $p_{\mathrm{T}}$ hadron are used to tag events to belong to a compared samples. The ALICE analysis uses $0.1 \%$ of the highest multiplicity $p p$ events for the classical ridge analysis. The results are compared to minimum-bias Pythia 8 predictions, a modified Pythia 8 simulation with strings shoving [11] and EPOS LHC [12] simulation that involves the hydrodynamical evolution of the system. Yields of particles in the ridge sector (distance between the particles forming the 2PC $1.6<|\Delta \eta|<1.8$ ) are shown in Fig. 3 . A moderate increase of that yield is observed with a jet and hadron momentum. It is reproduced moderately well by EPOS and Pythia 8 with string shoving. ALICE further studied the yield in the short-range away-side of the $2 \mathrm{PC}$ function as shown in Fig. 3 right side. Theory comparisons favour the EPOS LHC simulations.
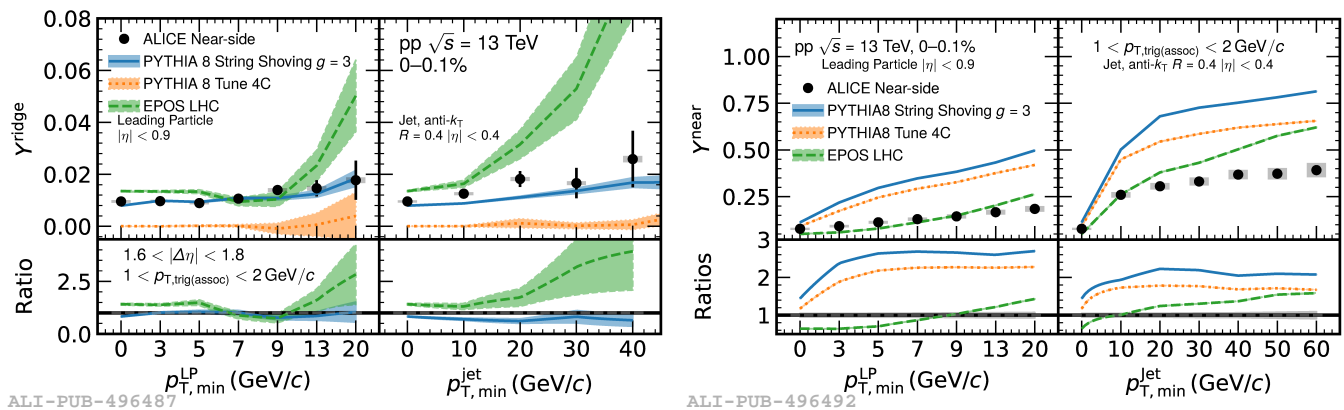

Figure 3: The relative yield of charged particles in the long-range near-side (left) and short-range away-side (right) parts of the $2 \mathrm{PC}$ as a function of the transverse momentum of the hardest jet or the hardest hadron in the event [10].

\section{Summary}

The collectivity of soft probes in collisions of the small systems is an area of intensive studies, both experimentally and theoretically. As shown in the analyses described, the modeling approaches that include the existence of QGP phase in the evolution tend to better represent the data. However, not all signatures of the QGP are observed in these collisions and further insights are needed in order to univocally confirm its presence. 


\section{References}

[1] ALICE Collaboration, The ALICE experiment at the CERN LHC, JINST 2 (2008) S08002.

[2] ATLAS Collaboration, The ATLAS Experiment at the CERN Large Hadron Collider, JINST 3 (2008) S08003.

[3] CMS Collaboration, The CMS experiment at the CERN LHC, JINST 3 (2008) S08004.

[4] CMS Collaboration, Observation of long-range, near-side angular correlations in protonproton collisions at the LHC, JHEP 1009 (2010) 091.

[5] CMS Collaboration, Elliptic Flow of Charm and Strange Hadrons in High-Multiplicity $p+P b$ Collisions at $\sqrt{s_{\mathrm{NN}}}=8.16 \mathrm{TeV}$, Phys. Rev. Lett. 121 (2018) 082301

[6] ATLAS Collaboration, Transverse momentum and process dependent azimuthal anisotropies in $\sqrt{s_{\mathrm{NN}}}=8.16 \mathrm{TeV} p+P b$ collisions with the ATLAS detector, Eur. Phys. J. C (2020) 8073

[7] ATLAS Collaboration, Measurement of long-range multiparticle azimuthal correlations with the subevent cumulant method in $p p$ and $p+P b$ collisions with the ATLAS detector at the CERN Large Hadron Collider, Phys. Rev. C 97 (2020) 024904

[8] ATLAS Collaboration, Measurement of long-range two-particle azimuthal correlations in Z-boson tagged pp collisions at $\sqrt{s}=8$ and 13 TeV, Eur. Phys. J. C (2020) 8064

[9] ATLAS Collaboration, Measurement of the sensitivity of two particle correlations in $p p$ collisions at $\sqrt{s}=13 \mathrm{TeV}$ to the presence of jets with the ATLAS detector, ATLAS-CONF2020-018

[10] ALICE Collaboration, Long- and short-range correlations and their event-scale dependence in high-multiplicity pp collisions at $\sqrt{s}=13 \mathrm{TeV}$, JHEP 05 (2021) 290

[11] Bierlich Ch., Gustafson G., Leif L., Collectivity without plasma in hadronic collisions, Phys. Let. B 779 (2018) 58

[12] Pierog T., Karpenko Iu., Katzy J.M., Yatsenko E., Werner K., EPOS LHC: Test of collective hadronization with data measured at the CERN Large Hadron Collider, Phys. Rev. C 92 (2015) 034906 